2. Lax, B., Mavroides, J.G., Zeiger, H.J., Keyes, R.I. (1960) Infrared magnetoreflection in bismuth // Phys. Rev. Lett. V. 5. № 6. 241-243.

3. McClure, J.W. (1976). The Energy Band Model for Bismuth: Resolution of a Theoretical Discrepancy. J. Low Temp. Phys, 25. № 5/6 (25). 527-540.

4. McClure, J.W., Choi, K. H. (1977). Energy band model and properties of electrons in bismuth. Solid State Communications, 21, 1015-1018.

5. Vasilyeva, I.I. (2019). Modelirovanie fizicheskogo processa v vismute s pomoshch'yu yazyka programmirovaniya PYTHON [Modeling the physical process in bismuth using the PYTHON programming language]. Materialy mezhdunarodnoj nauchno-prakticheskoj konferencii "Sistemy upravleniya, tekhnicheskie sistemy: ustojchivost', stabilizaciya, puti $i$ metody issledovaniya (24 aprelya 2019 g., Yelets) [Proceedings of international scientific-practical conference Control systems, technical systems: stability, stabilization, ways and methods of research (April 24, 2019, Yelets)], 134-138. (In Russ., abstract in Eng.)

6. Vasilyeva, I. I., Gladkikh, O. B. (2018). Podhod k nahozhdeniyu parametrov rasprostraneniya poverhnostnoj elektromagnitnoj volny na osnove chislennyh metodov modelirovaniya [An Approach to Finding The Parameters of Propagation of a Surface Electromagnetic Wave Based on Numerical Modeling Methods] Naukoemkie tekhnologii [Science-intensive technologies], 5(19), 49-55. (In Russ., abstract in Eng.)

7. Vasilyeva, I. I., Masina, O. N. (2014). Osobennosti issledovaniya zonnoj struktury vismuta [The Features of Investigation of Bismuth Band Structure] Mezhdunarodnyj akademicheskij vestnik [International Academic Bulletin], 1, 45-51. (In Russ., abstract in Eng.)

DOI: $10.24888 / 2500-1957-2021-1-69-80$

\begin{tabular}{c|l} 
УДК & ПОСТРОЕНИЕ ДИФФЕРЕНЦИАЛЬНЫХ \\
004.89, & МАТЕМАТИЧЕСКИХ МОДЕЛЕЙ, ИСПОЛЬЗУЕМЫХ ПРИ \\
37.022 & РАЗРАБОТКЕ ГИБРИДНОЙ ИНТЕЛЛЕКТУАЛЬНОЙ \\
& ОБУЧАЮЩЕЙ СРЕДЫ, С УЧЕТОМ ЗАПАЗДЫВАНИЯ И \\
& УПРАВЛЯЮЩИХ ВОЗДЕЙСТВИЙ
\end{tabular}

Дружинина Ольга Валентиновна

д.ф.-м.н., профессор

ovdruzh@mail.ru

г. Москва

Масина Ольга Николаевна

д.ф.-м.н., доцент

olga121@inbox.ru

г. Елец

Петров Алексей Алексеевич

к.т.н., ст. преподаватель

xeal91@yandex.ru

г. Елец
Федеральный исследовательский центр «Информатика и управление» Российской академии наук, Институт проблем управления им. В.А. Трапезникова Российской академии наук

Елецкий государственный университет им. И.А. Бунина

\footnotetext{
Аннотация. Направление, связанное с применением математического моделирования и интеллектуальных технологий в разработке методов и средств поддержки образовательных процессов, относится к числу актуальных научных направлений. Рассматриваются вопросы построения и анализа математических моделей, используемых при разработке гибридной
} 
интеллектуальной обучающей среды (ГИОС). При построении и исследовании моделей учитываются факторы запаздывания, управляющих воздействий и используются методы и алгоритмы интеллектуального анализа, в частности, методы нейросетевого моделирования. В рамках создания ГИОС предлагается построение дифференциальных математических моделей с запаздывающей обратной связью, а также дифференциальных моделей с управляющими воздействиями. Рассмотрена непрерывная динамическая модель, описывающая процесс получения и усвоения знаний, с учетом использования коэффициентов усвоения, мотивации ученика, уровня требований и управляющих воздействий в системе. Дана характеристика обобщенной модели образовательного процесса, содержащей модуль базы знаний и модуль взаимодействия на основе искусственного интеллекта, применительно к процессу изучения математики в системе общего образования. Для модели передачи заданий от учителя к ученику с учетом взаимодействия с ГИОС и с учетом запаздывающей по времени обратной связи изучен ряд качественных свойств. Описанный подход к анализу свойств моделей позволяет учитывать неопределенности и управляющие воздействия в гибридных обучающих средах. Охарактеризованы алгоритмы искусственного интеллекта и параметрической оптимизации, которые предполагают проведение эффективного численного анализа и вычислительных экспериментов, направленных на оценку процесса получения усвоения и контроля знаний по математике в системе общего образования.

Ключевые слова: гибридная интеллектуальная обучающая среда, дифференциальные модели, инструментально-методические средства поддержки образовательного процесса, устойчивость, стабилизация, нейросетевые алгоритмы, управляющие воздействия.

\section{Работа выполнена при финансовой поддержке РФФИ (проект № 19-29-14009).}

\section{Введение}

Синтез новых моделей, описывающих педагогические процессы, является необходимым этапом при создании гибридной интеллектуальной обучающей среды (ГИОС) в различных предметных областях, в том числе в предметной области, связанной с преподаванием математики в средней школе $[1,13,21]$. Цифровые математические образовательные платформы должны в перспективе предоставить инструменты для эффективной работы ученика, усовершенствовать средства организации среднего образования и оптимизировать учебное время. Использование современных цифровых технологий в образовательном процессе приводит, с одной стороны, к изменению программ и подходов к обучению, а с другой стороны - к разработке дополнительного инструментальнометодического обеспечения. Разрабатываемое инструментально-методическое обеспечение должно способствовать изучению с разных точек зрения процессов и явлений, учитывающих предметные особенности изучаемой дисциплины, психологические особенности и знания обучающихся. Кроме того, использование синергетического подхода к анализу процесса обучения позволяет заложить основы проектирования индивидуальных образовательных сред, складывающихся из образовательных элементов разных уровней с учетом процессов самоорганизации субъектов [22].

Вопросы синтеза и анализа математических моделей образовательного процесса рассматривались в $[7,9,11,12,17]$ и в других работах. Сущность основных направлений кибернетической педагогики и положения теории управления образовательными системами изложены в $[11,17]$. Следует отметить, что при построении и изучении моделей процесса 
обучения в рамках ГИОС представляет интерес следующее обобщение часто используемой постановки задачи: с учетом параметров учащихся, характеристик используемых методов учителя и используемой структуры ГИОС, учебной программы, оценить уровень знаний учеников в конце обучения. Для решения задач такого типа можно использовать методы имитационного моделирования $[11,12]$. Указанные методы в сочетании с методами теории оптимизации можно применить и к решению оптимизационных задач процесса обучения: найти распределение учебной информации, уровень требований учителя и оптимальную структуру соответствующего модуля ГИОС, при которых знания учеников в конце периода обучения достигнут заданного уровня с учетом того, что ограничения, накладываемые на процесс обучения, будут выполнены.

В $[11,12]$ предложены многокомпонентные модели обучения и рассмотрены вопросы их использования для исследования дидактических систем. Автором развивается информационно-кибернетический подход к анализу учебного процесса. Модели такого типа допускают эффективное использование методов компьютерного моделирования, позволяющих проводить серии компьютерных экспериментов с учетом различных начальных условий, параметров, внешних воздействий, а также позволяющих оценить состояние дидактической системы в конце обучения.

В настоящей работе изучены вопросы построения и анализа математических моделей, используемых в процессе разработки ГИОС. При построении и исследовании моделей учитываются факторы запаздывания, управляющие воздействий и используются методы и алгоритмы интеллектуального анализа, в частности, методы нейросетевого моделирования. Предлагается построение дифференциальных математических моделей с запаздывающей обратной связью, а также дифференциальных моделей с управляющими воздействиями. Рассмотрена непрерывная динамическая модель, описывающая процесс получения и усвоения знаний, с учетом использования коэффициентов усвоения, уровня требований и управляющих воздействий в системе. Дана характеристика обобщенной модели образовательного процесса, содержащей модуль базы знаний и модуль взаимодействия на основе искусственного интеллекта, применительно к процессу изучения математики в системе общего образования.

\section{Управляемые динамические модели с учетом запаздывания}

В векторном виде конечномерные динамические модели с учетом запаздывания и управляющих воздействий можно записать в следующем виде:

$$
\begin{gathered}
\dot{x}=g(t, x, \delta), \quad x \in R^{n}, \\
\dot{x}=g(t, x, \delta, u), \quad x \in R^{n},
\end{gathered}
$$

где $x \in R^{n}$ - вектор фазового состояния системы, $t$ - время, $\delta$ - вектор запаздывания, $u=u(x, t)-$ вектор управления.

Модели (1), (2) и их конкретизации используются при разработке одного из базовых модулей ГИОС - модуля построения моделей. К методам исследования указанных динамических моделей относятся, в частности, методы качественной теории динамических систем и теории управления $[3,16,20]$, а также методы численного анализа, параметрической оптимизации и интеллектуального анализа $[2,10,19,23]$. В частности, к числу важных задач можно отнести поиск состояний равновесия и анализ устойчивости в смысле Ляпунова относительно всех и части фазовых переменных применительно к неуправляемым моделям вида (1) и стабилизация применительно к управляемым моделям вида (2).

Рассмотрим случай управляемых динамических моделей (У ДМ). Для учета различных типов неопределенностей возможно осуществить переход от УДМ вида (2) к соответствующим УДМ, задаваемых дифференциальными включениями, нечеткими и стохастическими дифференциальными уравнениями (рис. 1). 


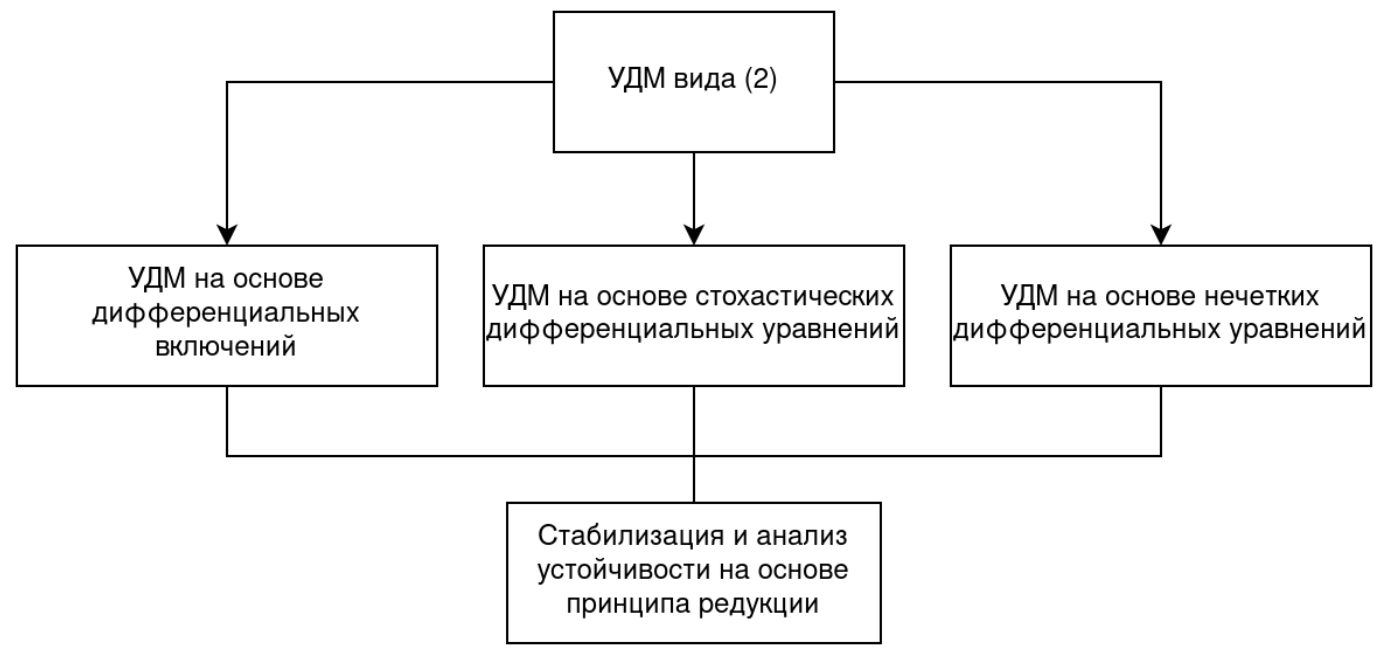

Рис. 1. Виды управляемых динамических моделей для описания образовательных процессов и иллюстрация единого подхода к анализу устойчивости и стабилизации

На основе принципа редукции задачи об устойчивости дифференциальных включений к задаче об устойчивости нечетких и стохастических дифференциальных уравнений формулируются конструктивные условия устойчивости и стабилизации $[5,14,20]$. Предлагается использовать указанный принцип применительно к моделям педагогического процесса, описываемых с помощью систем (1), (2). Следует отметить, к важным методам анализа относятся численные методы поиска траекторий систем (1), (2) и их частных случаев на основе модификаций и обобщений численных методов дифференциальных уравнений с применением прикладных математических пакетов.

Существуют различные подходы к оптимальному управлению системами вида (2), а именно, на основе управления с помощью ПИД-регуляторов [16] или с помощью введения системы в скользящие режимы [6]. Для получения оптимальных траекторий модели (2) можно использовать методы глобальной параметрической оптимизации (включая методы, инспирированные природой: нейросетевые методы, методы муравьиных колоний и др.) в сочетании с применением интеллектуальных технологий $[2,10,19,23]$.

\section{Модели многоступенчатой передачи заданий}

Рассмотрим модель многоступенчатой передачи учебных заданий, задаваемую дифференциальным уравнением $m$-го порядка, следующего вида:

$$
\frac{d^{m} x}{d t^{m}}=f(t, x(t), x(t-\delta t), \gamma(x, t)),
$$

где $x \in R^{n}$ - вектор фазового состояния системы, $t$ - время, $x(t-\delta t)$ - вектор запаздывания, $\gamma(x, t)-$ функция, задающая вектор возмущений в системе.

Обобщенную управляемую модель можно представить в виде:

$$
\frac{d^{n} x}{d t^{n}}=f(t, x(t), x(t-\delta t), \gamma(x, t), u(x, t))
$$

где часть входящих величин пояснена после уравнения (1), $u(x, t)$ - вектор управления.

В моделях (3) и (4) $n$-й порядок производной соответствует построению цепочки из $m$ звеньев передачи учебных заданий. В указанной цепочке присутствует качественная зависимость значения последующего звена от предыдущего. Системы (3), (4) задают непрерывные динамические модели с учетом запаздывания. Наличие вектора запаздывания обусловлено возникающими задержками при передаче заданий.

В модели (4) предполагается, что возмущения задаются функцией, на которую накладываются соответствующие ограничения. Для указанной модели можно поставить задачу оптимального управления с заданными начальными и граничными условиями. Задача 
оптимального управления заключается в нахождении управляющей функции $u(t, x)$, которая соответствует траектории с учетом выполнения заданных условий. В рамках этой задачи следует рассматривать фиксированный промежуток $\left(t_{1}, t_{2}\right)$. Кроме того, для модели (4) с учетом накладываемых условий также может рассматриваться постановка задачи быстродействия. Согласно такой постановке необходимо минимизировать значение разности $t_{2}$ и $t_{1}$. Следует также отметить, что интерес представляет также соответствующая задача оптимального управления со смешанными ограничениями [15].

Модель передачи заданий в цепочке «учитель - модуль ГИОС - ученик» с запаздывающей обратной связью и с возмущениями можно представить в виде:

$$
\begin{gathered}
\dot{x}_{1}=k_{1} x_{2}(t-\hat{t})+k_{5} x_{3}(t-2 \hat{t}), \\
\dot{x}_{2}=k_{2} x_{3}(t-\hat{t})+k_{6} x_{2}(t-\hat{t}), \\
\dot{x}_{3}=k_{3}+k_{4} x_{1}(t-3 \hat{t})+F_{1},
\end{gathered}
$$

где $x_{1}, x_{2}, x_{3}$ - взаимозависимые количественные показатели педагогического процесса для ученика, учителя и модуля ГИОС соответственно, $k_{1}, \ldots, k_{6}-$ коэффициенты параметров модели, $F_{1}$ - функция возмущения, $\hat{t}$ - единичная задержка в системе. Модель (5) является частным случаем модели (3), когда размерность модели равна 3.

Выполнены вычислительные эксперименты по нахождению траекторий модели (5). Результаты анализа трехмерной модели образовательного процесса показывают, что при заданном наборе начальных значений и значений параметров поведение траекторий имеет характер осцилляций [21]. Серия компьютерных экспериментов демонстрирует аналогичное поведение траекторий. Перспективным направлением является изучение управляемой трехмерной модели и построение регуляторов на основе методов глобальной параметрической оптимизации.

Синтез и анализ модели (5) выполняется с учетом некоторых допущений. В частности, предполагается, что процесс передачи заданий является непрерывным. Для получения уточняющих результатов планируется использование комплексных методик моделирования, в частности, моделей системной динамики и мультиагентных систем.

\section{Модели образовательного процесса с учетом прочности усвоения получаемых знаний}

В $[11,12]$ рассмотрены многокомпонентные модели образовательного процесса, в которых принимается во внимание различие в скорости забывания различных элементов учебного материала и переход непрочных знаний в разряд прочных. При этом учитывается, что процесс усвоения и запоминания учебной информации связан с установлением ассоциативных связей между новыми и уже имеющимися знаниями. В результате многократного использования формируются навыки, которые сохраняются длительное время, а приобретенные знания становятся более прочными, при этом элементы учебного материала разделены на несколько категорий с учетом разной прочности знаний.

Будем использовать ряд обозначений согласно [12]. Пусть $U$ - уровень требований, предъявляемый учителем и согласованный с соответствующим модулем ГИОС. Для двухкомпонентной модели предполагается, что суммарные знания ученика состоят из знаний двух категорий: $Z=z_{1}+z_{2}$, где $z_{1}$ - менее прочные знания с более высоким коэффициентом абывания $\gamma_{1}, z_{2}$ - более прочные знания с более низким коэффициентом забывания $\gamma_{2}$. Коэффициент $\kappa$ является индикатором процесса: пока происходит обучение $\kappa=1$, когда же обучение прекращается, $\kappa=0$. Через $\alpha_{i}$ обозначены коэффициенты усвоения знаний. Двухкомпонентная модель образовательного процесса задается системой обыкновенных дифференциальных уравнений вида:

$$
\begin{gathered}
\dot{z}_{1}=\kappa \alpha_{1}(U-Z)-\kappa \alpha_{2} z_{1}-\gamma_{1} z_{1}, \\
\dot{z}_{2}=\kappa \alpha_{2} z_{1}-\gamma_{2} z_{2} .
\end{gathered}
$$


Для модели $(6)$ в $[11,12]$ изложены результаты имитационного моделирования, в ходе которого учитывался линейный рост уровня требований: $U=a\left(t-t_{0}\right)+b$. В этих работах построены и изучены также многокомпонентные модели для $n$-мерного случая, когда рассматривается $n$ категорий знаний.

Рассмотрим обобщение модели (6) на управляемый случай

$$
\begin{gathered}
\dot{z}_{1}=\kappa \alpha_{1}(U-Z)-\kappa \alpha_{2} z_{1}-\gamma_{1} z_{1}+u_{1}, \\
\dot{z}_{2}=\kappa \alpha_{2} z_{1}-\gamma_{2} z_{2}+u_{2},
\end{gathered}
$$

где $u_{i}(i=1,2)-$ функции управления.

С помощью задания критериев качества управления образовательным процессом можно построить управляющие функции, при которых решаются такие задачи, как поиск оптимального уровня требований и оптимального распределения учебной информации, при этом достигается максимально возможный уровень знаний в конце периода обучения. Для решения задач оптимального управления для модели (7) и ее обобщений на более высокую размерность можно использовать методы теории оптимального управления, методы глобальной параметрической оптимизации, нейросетевые методы, методы построения нечетких регуляторов, методы машинного обучения.

\section{Структура модели процесса обучения с применением искусственного интеллекта и базы знаний}

Важным вопросом разработки гибридной обучающей среды является анализ предметной области. Если рассматривать образовательный процесс как единую систему, то сложность процесса заключается в наличии объективных и субъективных факторов. Для построения базы знаний (Б3) гибридной интеллектуальной обучающей среды (ГИОС) на основе технологий искусственного интеллекта (ИИ) помимо дидактических материалов необходимо наличие исходных данных, репрезентативно отражающих основные закономерности функционирования образовательной системы. Структура модели образовательного процесса применительно к процессу обучения математике в системе общего образования с учетом построения БЗ и алгоритмов ИИ представлена на рис. 2.

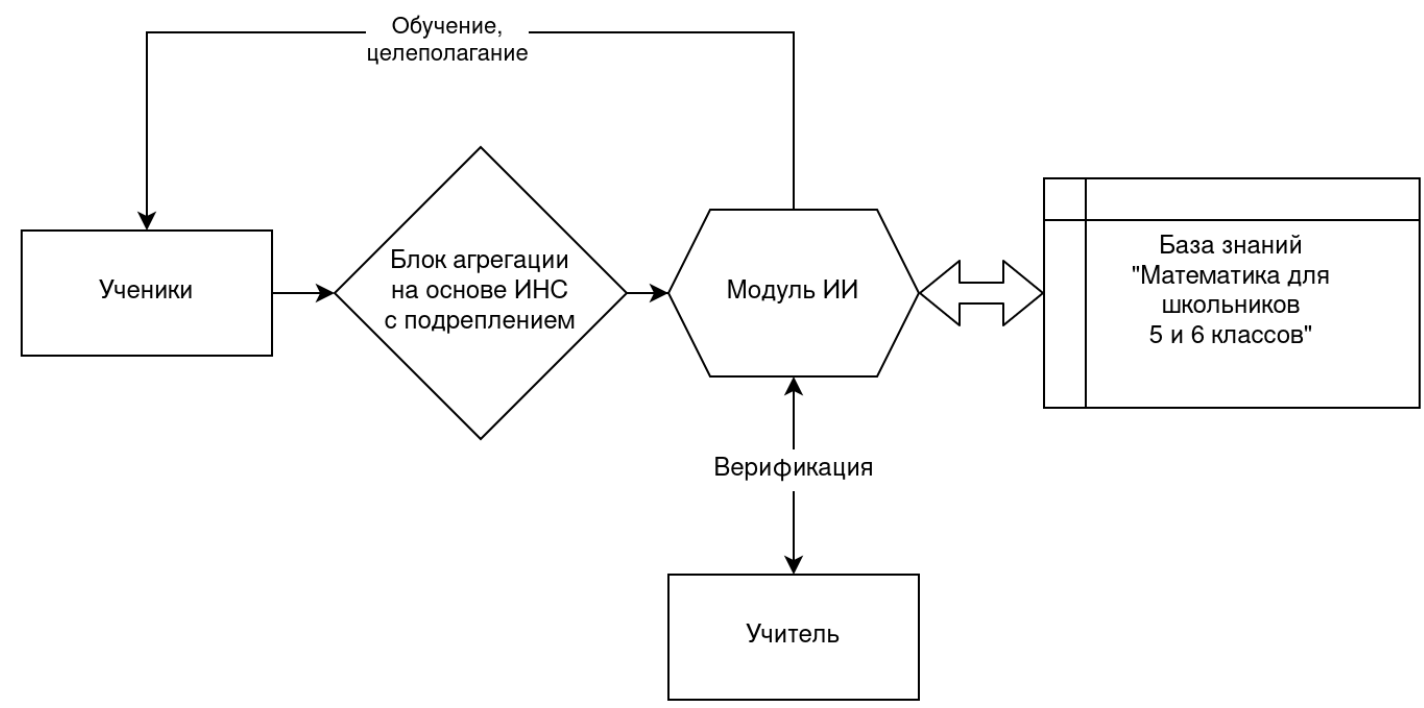

Рис. 2. Структура обобщенной модели образовательного проиесса с использованием модулей ИИ и БЗ в рамках разработки ГИОС (на примере обучения математике в системе общего образования)

Отметим, что блок агрегации имеет вариативность в зависимости от используемых нейросетевых алгоритмов. В частности, можно использовать нейросети с различным количеством слоев, нейросети с задержкой, нейросети с подкреплением. В частности, в [24] 
предлагается использовать нейронную сеть 2-3 скрытых слоев с идентичным числом нейронов (по 10) с выходной фиксацией состояния нейрона по мере прохождения по скрытым слоям.

В рамках создания ГИОС при решении актуальных задач образовательного процесса, в частности, для анализа построенных дифференциальных математических моделей с запаздывающей обратной связью и с управлением, могут использоваться методы нейросетевого моделирования, предложенные в [18]. Вопросы, связанные с изучением устойчивости сложных систем, к которым относятся и гибридные обучающие системы, могут быть исследованы с помощью результатов $[4,8]$, в которых содержатся, в частности, методы анализа систем интеллектного управления. Существуют различные подходы к получению исходных данных, в частности, анализ и синтез, натурный эксперимент и вычислительный эксперимент.

Анализ и синтез имеют ограниченную применимость из-за специфики предметной области. Натурный эксперимент можно считать достаточно эффективным, однако его организация является наиболее трудоемкой. Вычислительный эксперимент сочетает в себе преимущества первого и второго подхода - он может быть выполнен исследователем самостоятельно, и при этом его результаты могут отражать скрытые закономерности изучаемого процесса.

В рамках дальнейшего изучения моделей (5) и (7) представляет интерес построение конкретных критериев качества управления, оптимизирующих процессы передачи заданий и получения знаний. Для нахождения управляющих функций с учетом начальных значений переменных и заданных наборов параметров можно использовать методы глобальной параметрической оптимизации [10] в сочетании с методами нейросетевого моделирования [19] и с другими методами. Предлагается использовать разработанные нейросетевые алгоритмы и создать модули программного комплекса моделирования систем, обеспечивающих функционирование гибридной обучающей среды с применением современных подходов к моделированию.

Важно отметить, что теоретический и практический интерес представляет стабилизация до асимптотической устойчивости моделей вида (2), (4), (5), (7) и анализ возможностей решения линейных матричных неравенств при аппроксимации с помощью ТСмоделей $[25,26]$. Применение аппарата ТС-моделей позволяет использовать современные вычислительные системы для решения линейных матричных неравенств, описывать свойства исходных нелинейных систем и решать задачи устойчивости и стабилизации.

\section{Заключение}

Таким образом, в настоящей работе изучены вопросы построения и интеллектуального анализа моделей образовательного процесса в рамках разработки ГИОС. Рассмотрены обобщенные управляемые динамические модели с учетом запаздывания. В качестве конкретизаций изучены модели многоступенчатой передачи заданий и модели образовательного процесса с учетом прочности усвоения полученных знаний. Описана структура модели процесса обучения с применением искусственного интеллекта и базы знаний. Предлагаемые методы решения задач устойчивости и оптимального управления педагогическим процессом в условиях неопределенности демонстрируют достаточно высокий уровень формализуемости и применимости инструментов искусственного интеллекта. Полученные результаты могут найти применение при разработке гибридной обучающей среды с использованием искусственного интеллекта, а также при решении задач оптимального управления моделями образовательных и социально-экономических систем.

Описанный подход к анализу свойств моделей позволяет учитывать неопределенности и управляющие воздействия в гибридных обучающих средах. Охарактеризованы алгоритмы искусственного интеллекта и параметрической оптимизации, которые предполагают проведение эффективного численного анализа и вычислительных экспериментов, направленных на оценку процесса получения усвоения и контроля знаний по математике в системе общего образования. 


\section{Список литературы}

1. Басалин П.Д., Тимофеев А.Е., Кумагина Е.А., Неймарк Е.А., Фомина И.А., Чернышова Н.Н. Реализация гибридной интеллектуальной обучающей среды продукционного типа // Современные информационные технологии и ИТ-образование. 2018. Т. 14. № 1. C. 256-267.

2. Васильев В.И., Ильясов Б.Г. Интеллектуальные системы управления. Теория и практика. М.: Радиотехника, 2009.

3. Демидович Б.П. Математическая теория устойчивости. М.: Наука, 1967.

4. Дружинина О.В., Масина О.Н. Методы анализа устойчивости систем интеллектного управления. М.: Изд. группа URSS, 2016.

5. Дружинина О.В., Масина О.Н. Методы исследования устойчивости и управляемости нечетких и стохастических динамических систем. М.: ВЦ РАН, 2009.

6. Емельянов С.В., Коровин С.К., Левант А. Скользящие режимы высших порядков в системах управления // Дифференц. уравнения. 1993. Т. 29. №11. С. 1877-1899.

7. Ивашкин Ю.А., Назойкин Е.А. Мультиагентное имитационное моделирование процесса накопления знаний // Программные продукты и системы. 2011. №1 . С. 47-52.

8. Игонина О.В., Масина О.Н., Дружинина О.В. Анализ устойчивости динамических систем на основе методов интеллектного управления и свойств линейных матричных неравенств. Монография. Елец: ЕГУ им. И.А. Бунина, 2020.

9. Леонтьев Л.П., Гохман О.Г. Проблемы управления учебным процессом: Математические модели. Рига: Зинатне, 1984.

10. Карпенко А.П. Современные алгоритмы поисковой оптимизации. Алгоритмы, вдохновленные природой. М.: МГТУ им. Н.Э. Баумана, 2017.

11. Майер Р.В. Кибернетическая педагогика: имитационное моделирование процесса обучения. Глазов: Глазовский государственный педагогический институт, 2014.

12. Майер Р.В. Многокомпонентная модель обучения и ее использование для исследования дидактических систем // Фундаментальные исследования. 2013. №10. С. 2524-2528.

13. Масина О.Н., Дружинина О.В., Петров А.А. Построение и анализ моделей педагогического процесса в условиях неопределенности // Материалы Международной научной конференции «Фундаментальные проблемы обучения математике, информатике и информатизации образования», посвященной 180-летию педагогического образования в г. Ельце (Елец, ЕГУ им. И.А. Бунина, 25-27 сентября 2020 г.). Елец: ЕГУ им. И.А. Бунина, 2020. С. 87-89.

14. Меренков Ю.Н. Устойчивоподобные свойства дифференциальных включений, нечетких и стохастических дифференциальных уравнений. М.: Изд-во РУДН, 2000.

15. Милютин А.А., Дмитрук А.В., Осмоловский Н.П. Принцип максимума в оптимальном управлении. М.: Изд-во МГУ, 2004.

16. Никулин Е. А. Основы теории автоматического управления. Частотные методы анализа и синтеза систем. СПб.: БХВ-Петербург, 2004.

17. Новиков Д.А. Теория управления образовательными системами. М.: Народное образование, 2009.

18. Петров А.А., Масина О.Н., Дружинина О.В. Особенности применения интеллектуальных технологий в разработке базового математического и программного обеспечения гибридной обучающей среды // Материалы VI Международной научнопрактической конференции «Системы управления, сложные системы: моделирование, устойчивость, стабилизация, интеллектуальные технологии», посвященной 100 -летию со дня рождения профессора А.А. Шестакова (Елец, 16-17 сентября 2020 г.). Елец: ЕГУ им. И.А. Бунина, 2020. С. 69-71.

19. Тархов Д.А. Нейросетевые модели и алгоритмы. М.: Радиотехника, 2014.

20. Шестаков А.А. Обобщенный прямой метод Ляпунова для систем с распределенными параметрами. М.: Наука, 1990. 2-е изд. доп. М.: URSS, 2007. 
21. Druzhinina O.V., Masina O.N., Petrov A.A., Shcherbatykh S.V. Application of intelligent technologies and neural network modeling methods in the development of a hybrid learning environment // Journal of Physics: Conference Series. 2020. V. 1691. P. 012125(1-6).

22. Dvoryatkina S., Shcherbatykh S., Shcherbatykh L. Synergy of mathematics, informatics and innovative didactics (on the example of retraining of teachers of mathematics) // Proceedings of the 12th International Conference on Education and New Learning Technologies (ICERI2018), 12th-14th November, 2018. Seville, Spain. P. 2503-2509.

23. Price K., Storn R., Lampinen J. Differential Evolution: A Practical Approach to Global Optimization. Springer, 2005.

24. Smirnov E.I., Shcherbatykh S.V. Parameters and classifier of neural network databases of educational results // Материалы Международной научной конференции «Фундаментальные проблемы обучения математике, информатике и информатизации образования», посвященной 180-летию педагогического образования в г. Ельце (Елец, ЕГУ им. И.А. Бунина, 25-27 сентября 2020 г.). Елец: ЕГУ им. И.А. Бунина, 2020. C. $162-163$.

25. Takagi T., Sugeno M. Fuzzy identification of systems and its appli-cations to modeling and control // IEEE Trans. Syst., Man and Cybernetics. 1985, 15, 116-132.

26. Tanaka K., Wang H.O. Fuzzy control systems design and analysis: a linear matrix inequality approach. N.Y.: Wiley, 2001.

\title{
CONSTRUCTION OF DIFFERENTIAL MATHEMATICAL MODELS USED IN THE DEVELOPMENT OF A HYBRID INTELLECTUAL LEARNING ENVIRONMENT, TAKING INTO ACCOUNT DELAY AND CONTROL ACTIONS
}

\author{
O.V. Druzhinina \\ Dr. Sci. (Physics \& Math.), professor \\ ovdruzh@mail.ru \\ Moscow \\ Federal Research Center «Computer Science \\ and Control» of Russian Academy of Sciences, \\ V.A. Trapeznikov Institute of Control Sciences \\ of Russian Academy of Sciences \\ O.N. Masina \\ Bunin Yelets State University \\ Dr. Sci. (Physics \& Math.), associate \\ professor \\ olga121@inbox.ru \\ Yelets \\ A.A. Petrov \\ Ph.D. (Tech), Senior Lecturer \\ xeal91@yandex.ru \\ Yelets
}

\begin{abstract}
The direction associated with the use of mathematical modeling and intelligent technologies in the development of methods and means of supporting educational processes is one of the relevant scientific areas. The issues of construction and analysis of mathematical models used in the development of a hybrid intelligent learning environment (GILE) are considered. When constructing and researching models, factors of delay, control actions are taken into account, and methods and algorithms of intelligent analysis are used, in particular, methods of neural network modeling. As part of the creation of GILE, it is proposed to build differential mathematical models with delayed feedback, as well as differential models with control actions. A continuous dynamic model is considered that describes the process
\end{abstract}


of obtaining and assimilating knowledge, taking into account the use of assimilation coefficients, student motivation, the level of requirements and control actions in the system. A characteristic is given of a generalized model of the educational process, containing a knowledge base module and an interaction module based on artificial intelligence, in relation to the process of studying mathematics in the general education system. For the model of transferring tasks from teacher to student, taking into account the interaction with the GIOS and taking into account the time-delayed feedback, a number of qualitative properties have been studied. The described approach to the analysis of model properties makes it possible to take into account uncertainties and control actions in hybrid learning environments. Algorithms of artificial intelligence and parametric optimization, which imply effective numerical analysis and computational experiments aimed at assessing the process of obtaining the assimilation and control of knowledge in mathematics in the general education system, are characterized.

Keywords: hybrid intelligent learning environment, differential models, instrumental and methodological means of supporting the educational process, stability, stabilization, neural network algorithms, control actions.

\section{References}

1. Basalin, P.D., Timofeev, A.E., Kumagina, E.A., Neymark, E.A., Fomina, I.A., Chernyshova, N.N. (2018) Realizatsiya gibridnoy intellektual'noy obuchayushchey sredy produktsionnogo tipa [Implementation of a hybrid intelligent production-type learning environment]. Sovremennye informatsionnye tekhnologii $i$ IT-obrazovanie [Modern information technology and IT-education], 14(1), pp. 256-267. (In Russ., abstract in Eng.)

2. Demidovich, B.P. (1967) Matematicheskaya teoriya ustoychivosti [Mathematical theory of stability]. Moscow: The science. (In Russ.)

3. Druzhinina, O.V., Masina, O.N. (2016) Metody analiza ustoychivosti sistem intellektnogo upravleniya [Methods for analyzing the stability of intelligent control systems]. Moscow: URSS. (In Russ.)

4. Druzhinina, O.V., Masina, O.N. (2009) Metody issledovaniya ustoychivosti i upravlyaemosti nechetkikh $i$ stokhasticheskikh dinamicheskikh system [Methods for studying the stability and controllability of fuzzy and stochastic dynamical systems]. Moscow: Dorodnitsyn Computing Center of RAS. (In Russ.)

5. Druzhinina, O.V., Masina, O.N., Petrov, A.A., Shcherbatykh, S.V. (2020). Application of intelligent technologies and neural network modeling methods in the development of a hybrid learning environment [Primenenie intellektual'nyh tehnologij i metodov nejrosetevogo modelirovanija pri razrabotke gibridnoj obuchajushhej sredy]. Journal of Physics: Conference Series, 1691, 012125(1-6). (In Eng.)

6. Dvoryatkina, S., Shcherbatykh, S., Shcherbatykh, L. (2018) Synergy of mathematics, informatics and innovative didactics (on the example of retraining of teachers of mathematics) [Sinergija matematiki, informatiki i innovacionnoj didaktiki (na primere perepodgotovki uchitelej matematiki)] In: Proceedings of the 12th International Conference on Education and New Learning Technologies (ICERI-2018), 12th-14th November, 2018 (pp. 2503-2509). Seville, Spain. (In Eng.)

7. Emel'yanov, S.V., Korovin, S.K., Levant, A. (1993) Skol'zyashchie rezhimy vysshikh poryadkov $\mathrm{v}$ sistemakh upravleniya [Higher-order sliding modes in control systems] Differents. Uravneniya [Differential Equations], 29(11), pp. 1877-1899. (In Russ.)

8. Igonina, O.V., Masina, O.N., Druzhinina, O.V. (2020) Analiz ustoychivosti dinamicheskikh sistem na osnove metodov intellektnogo upravleniya $i$ svoystv lineynykh matrichnykh neravenstv. Monografiya [Analysis of the stability of dynamic systems based on intelligent 
control methods and properties of linear matrix inequalities. Monograph]. Yelets: Bunin Yelets State University. (In Russ.)

9. Ivashkin, Yu.A., Nazoykin, E.A. (2011) Mul'tiagentnoe imitatsionnoe modelirovanie protsessa nakopleniya znaniy [Multi-agent simulation modeling of the knowledge accumulation process]. Programmnye produkty i sistemy [Software products and systems], 1, pp. 47-52. (In Russ.)

10. Karpenko, A.P. (2017) Sovremennye algoritmy poiskovoy optimizatsii. Algoritmy, vdokhnovlennye prirodoy [Modern search engine optimization algorithms. Algorithms inspired by nature]. Moscow: Moscow State Technical University. (In Russ.)

11. Leont'ev, L.P., Gokhman, O.G. (1984) Problemy upravleniya uchebnym protsessom: Matematicheskie modeliyu [Educational process management problems: Mathematical models]. Riga: Zinatne. (In Russ.)

12. Masina, O.N., Druzhinina, O.V., Petrov, A.A. (2020) Postroenie i analiz modeley pedagogicheskogo protsessa $\mathrm{v}$ usloviyakh neopredelennosti [Construction and analysis of models of the pedagogical process in conditions of uncertainty]. Materialy Mezhdunarodnoy nauchnoy konferentsii «Fundamental'nye problemy obucheniya matematike, informatike $i$ informatizatsii obrazovaniya», posvyashchennoy 180-letiyu pedagogicheskogo obrazovaniya $v$ g. El'tse (25-27 sentyabrya 2020 g., Elets). [Proceedings of the International Scientific Conference "Fundamental Problems of Teaching Mathematics, Informatics and Informatization of Education" dedicated to the 180th anniversary of pedagogical education in Yelets (September 25-27, 2020, Yelets)], pp. 87-89. (In Russ.)

13. Mayer, R.V. (2013) Mnogokomponentnaya model' obucheniya i ee ispol'zovanie dlya issledovaniya didakticheskikh system [Multicomponent learning model and its use for the study of didactic systems] Fundamental'nye issledovaniya [Fundamental Research], 10, 2524-2528. (In Russ.)

14. Mayer, R.V. (2014) Kiberneticheskaya pedagogika: imitatsionnoe modelirovanie protsessa obucheniya [Cybernetic Pedagogy: Simulation of the Learning Process]. Glazov. (In Russ.)

15. Merenkov, Yu.N. (2000) Ustoychivopodobnye svoystva differentsial'nykh vklyucheniy, nechetkikh $i$ stokhasticheskikh differentsial'nykh uravneniy [Stable-like properties of differential inclusions, fuzzy and stochastic differential equations]. Moscow: RUDN Publishing House. (In Russ.)

16. Milyutin, A.A., Dmitruk, A.V., Osmolovskiy N.P. (2004) Printsip maksimuma v optimal'nom upravlenii [The maximum principle in optimal control]. Moscow: Publishing house of Moscow State University. (In Russ.)

17. Nikulin, E.A. (2004) Osnovy teorii avtomaticheskogo upravleniya. Chastotnye metody analiza i sinteza system [Foundations of the theory of automatic control. Frequency methods of analysis and synthesis of systems]. St. Petersburg: BHV -Peterburg. (In Russ.)

18. Novikov, D.A. (2009) Teoriya upravleniya obrazovatel'nymi sistemami [Control theory of educational systems]. Moscow: Public education.

19. Petrov, A.A., Masina, O.N., Druzhinina, O.V. (2020) Osobennosti primeneniya intellektual'nykh tekhnologiy $\mathrm{v}$ razrabotke bazovogo matematicheskogo i programmnogo obespecheniya gibridnoy obuchayushchey sredy [Features of the use of intelligent technologies in the development of basic mathematical and software for a hybrid learning environment] Materialy VI Mezhdunarodnoy nauchno-prakticheskoy konferentsii "Sistemy upravleniya, slozhnye sistemy: modelirovanie, ustoychivost', stabilizatsiya, intellektual'nye tekhnologii», posvyashchennoy 100-letiyu so dnya rozhdeniya professora A.A. Shestakova (16-17 sentyabrya 2020 g., Elets). [Proceedings of the VI International Scientific and Practical Conference "Control Systems, Complex Systems: Modeling, Stability, Stabilization, Intelligent Technologies" dedicated to the 100th anniversary of the birth of Professor A.A. Shestakova (September 16-17, 2020, Yelets), pp. 69-71. (In Russ.) 
20. Price, K., Storn, R., Lampinen, J. (2005) Differential Evolution: A Practical Approach to Global Optimization [Differencial'naja jevoljucija: prakticheskij podhod $k$ global'noj optimizacii]. Springer. (In Eng.)

21. Shestakov, A.A. (2007) Obobshchennyy pryamoy metod Lyapunova dlya sistem $s$ raspredelennymi parametrami [Generalized direct Lyapunov method for systems with distributed parameters]. Moscow: URSS. (In Russ.)

22. Smirnov, E.I., Shcherbatykh, S.V. (2020) Parametry i klassifikator nejrosetevyh baz obrazovatel'nyh rezul'tatov [Parameters and classifier of neural network databases of educational results] Materialy Mezhdunarodnoy nauchnoy konferentsii «Fundamental'nye problemy obucheniya matematike, informatike $i$ informatizatsii obrazovaniya», posvyashchennoy 180-letiyu pedagogicheskogo obrazovaniya $v$ g. El'tse (25-27 sentyabrya 2020 g., Elets). [Proceedings of the International Scientific Conference "Fundamental Problems of Teaching Mathematics, Informatics and Informatization of Education" dedicated to the 180th anniversary of pedagogical education in Yelets (September 25-27, 2020, Yelets)], pp. 162-163. (In Eng.)

23. Takagi, T., Sugeno, M. (1985) Fuzzy identification of systems and its applications to modeling and control [Nechetkaja identifikacija sistem i ee prilozhenija $k$ modelirovaniju $i$ upravleniju]. IEEE Trans. Syst., Man and Cybernetics, 15, 116-132. (In Eng.)

24. Tanaka, K., Wang, H.O. (2001) Fuzzy control systems design and analysis: a linear matrix inequality approach [Razrabotka $i$ analiz sistem nechetkogo upravlenija: podhod $s$ ispol'zovaniem linejnogo matrichnogo neravenstva]. N.Y.: Wiley. (In Eng.)

25. Tarkhov, D.A. (2014) Neyrosetevye modeli $i$ algoritmy [Neural network models and algorithms]. Moscow: Radio engineering. (In Russ.)

26. Vasil'ev, V.I., Il'yasov, B.G. (2009) Intellektual'nye sistemy upravleniya. Teoriya i praktika [Intelligent control systems. Theory and practice]. Moscow: Radio engineering. (In Russ.)

DOI: $10.24888 / 2500-1957-2021-1-80-88$

\title{
УДК $\mid$ ТЕСТ ГИЛЕВА И МЕТОДИКА АРDЕХ КАК СРЕДСТВА 004.424.22 ТЕСТИРОВАНИЯ ПЛАТФОРМ 1С
}

\author{
Иванников Илья Сергеевич $\mid$ Елецкий государственный университет \\ аспирант им. И.А. Бунина \\ mail@yandex.ru \\ г. Елец
}

\begin{abstract}
Аннотация. В статье рассматривается базовая методика анализа проблем производительности в работающей многопользовательской системе 1С:Предприятие 8. Проблемы производительности не локализованы в определенных бизнес-процессах, а "равномерно распределены" по всей функциональности системы. В связи с этим внедрение новой платформы 1С на предприятии, необходимо не только провести тестирование нового функционала, убедиться в работоспособности старого, но и быть готовым к увеличению выделяемых ресурсов или апгрейду серверов. С каждой новой версией платформы 1C аппаратных ресурсов нужно больше, чтобы сохранить производительность и быстродействие системы как минимум на том же уровне.
\end{abstract}

Ключевые слова: платформа, релиз, методика APDEX, тест Гилева. 